\title{
A case of endocarditis: When three equals one
}

\author{
Kiran Kaur Kundhal MD, Andrew Mark Morris MD MSc FRCPC
}

\begin{abstract}
$\mathrm{A}^{32}$ 32 year-old male smoker presented to the emergency room with increasing breathlessness over two weeks. Past history was unremarkable, including no history of rheumatic fever, heart murmurs, intravenous drug use or recent invasive procedures. Oral hygiene was poor, and an aortic insufficiency murmur was heard on physical examination. Blood work revealed leukocytosis (white blood cells $=18.3 \times 10^{9} / \mathrm{L}$ ), hyperglycemia (serum blood glucose $=17.8 \mathrm{mmol} / \mathrm{L}$ ) and ketoacidosis. A chest radiograph displayed cardiomegaly, pulmonary edema and bilateral pneumonia. Serial electrocardiograms showed ST elevation in the precordial leads and troponin I was $4 \mu \mathrm{g} / \mathrm{L}$. Two initial blood cultures grew coagulase-negative staphylococci (CNS). The patient was treated with levofloxacin and vancomycin, along with usual therapy for hyperglycemia, congestive heart failure and acute coronary syndrome. A transesophageal echocardiogram (TEE) revealed severe aortic insufficiency, an aortic root abscess and a large cauli-
\end{abstract}

flower-shaped vegetation extending into the aortic valve. The patient underwent urgent aortic valve replacement and pericardial closure of the abscess. Two preoperative blood cultures each grew Staphylococcus warneri and Staphylococcus haemolyticus, as speciated by VITEK (bioMerieux, USA). Intraoperative aortic valve and pericardial fluid cultures, as well as two postoperative blood cultures, grew Staphylococcus epidermidis and S warneri, which were also speciated by VITEK. The automated antibiograms produced by VITEK for the various isolates differed, which is consistent with a polymicrobial endocarditis. The patient was treated with vancomycin, rifampin and gentamicin (to which all isolates were sensitive), but worsened clinically and demonstrated persistent fever.

What is your diagnosis? What would you recommend?

continued on page 405 
continued from page 400

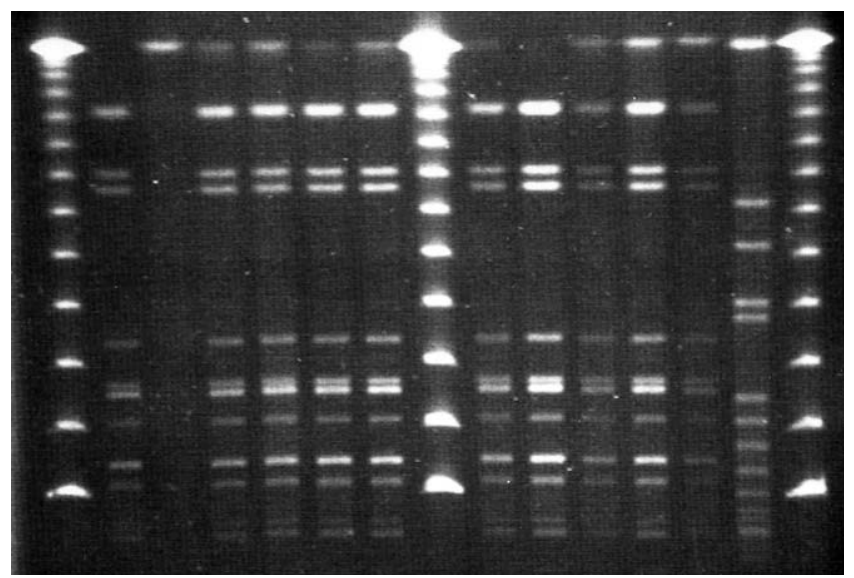

Figure 1) Pulsed-field gel electrophoresis demonstrating identical electrophoretic patterning in coagulase-negative staphylococci isolates from pericardial fluid (lane 2), aortic valve vegetation (lanes 3 and 4) and blood (lanes 5-7, 9-13). Lanes 1, 8 and 15 are markers, and lane 14 is a negative control

\section{DIAGNOSIS}

Follow-up TEE demonstrated increased vegetation size and new mitral regurgitation. The patient was taken back to the operating room for aortic and mitral valve replacements two-and-a-half weeks after his initial aortic valve replacement. Pulsed-field gel electrophoresis (PFGE) demonstrated identical electrophoretic patterning for all previous bacterial isolates, which was consistent with a monomicrobial CNS native-valve endocarditis (Figure 1). The patient recovered with a six-week course of vancomycin, gentamicin and rifampin. The regional reference laboratory was unable to speciate all of the isolates.

\section{DISCUSSION}

This patient presented with a case of native-valve endocarditis with CNS. He had no history of valvular surgery, intravenous drug use or percutaneous vascular access. CNS usually cause prosthetic-valve endocarditis and are uncommon causes of native-valve endocarditis, accounting for less than $5 \%$ of such cases.

Native-valve CNS endocarditis is primarily a community-acquired infection. Most case series have demonstrated an underlying cardiac abnormality, either congenital or acquired, in $65 \%$ to $75 \%$ of patients $(1,2)$. Clinical presentation often involves weight loss, weakness and fever, and up to one-third of patients may exhibit peripheral manifestations, including Osler's nodes, Janeway lesions and splinter hemorrhages (2). Common laboratory abnormalities include anemia and leukocytosis. In approximately one-half of patients, vegetations may be demonstrated via transtho- racic echocardiography (2). Mortality is between $13 \%$ and $20 \%(1,3,4)$.

At least $18 \mathrm{CNS}$ species have been isolated from human skin (5). S epidermidis accounts for nearly three-quarters of all isolates (6). However, it has been postulated that $S$ epidermidis possesses virulence factors that are not characteristic of other CNS (7). Other clinically significant species include Staphylococcus saprophyticus, Staphylococcus hominis, S haemolyticus, S warneri and Staphylococcus simulans.

Because polymicrobial endocarditis is uncommon in noninjection drug users or persons without indwelling vascular catheters, PFGE was performed to verify the varying antibiogram results in this case. Discovered in 1984, PFGE is a molecular technique of separating complex mixtures of DNA into different sized fragments by electrophoresis. DNA is isolated intact and is then treated with restriction enzymes to generate pieces small enough to resolve by electrophoresis in agarose or acrylamide. PFGE allows extremely large DNA to be resolved, with the upper size limit of DNA being well over $10 \mathrm{Mb}(10,000 \mathrm{~kb})$. During continuous field electrophoresis, DNA that is 30 to $50 \mathrm{~kb}$ migrates with the same mobility regardless of size. This is seen in a gel as a single large diffuse band. During PFGE, DNA is forced to change direction during electrophoresis so that different sized fragments begin to separate from each other. Smaller DNA fragments move in the new direction more quickly than larger DNA fragments with each reorientation of the electric field relative to the gel. Thus, larger DNA fragments lag behind smaller ones, providing a visible separation.

PFGE is particularly useful in apparent CNS infections to help differentiate true pathogens from sterile site contaminants $(8,9)$. Repetitive isolation of the same strain from an individual patient increases the probability of clinical significance.

In our case, PFGE demonstrated an identical electrophoretic pattern for all previous bacterial isolates, consistent with a monomicrobial CNS native-valve endocarditis. We recommend that clinicians be skeptical of apparent polymicrobial endocarditis in persons without percutaneous vascular access and that they also recognize the clinical role of molecular techniques to aid diagnosis and therapy in infectious diseases.

\section{REFERENCES}

1. Caputo GM, Archer GL, Calderwood SB, DiNubile MJ Karchmer AW. Native valve endocarditis due to coagulase-negative staphylococci. Clinical and microbiologic features. Am J Med 1987;83:619-25.

2. Whitener C, Caputo GM, Weitekamp MR, Karchmer AW. Endocarditis due to coagulase-negative staphylococci. Microbiologic, epidemiologic and clinical considerations. Infect Dis Clin North Am 1993;7:81-96.

3. Williams DN, Peterson PK, Verhoef J, Laverdiere M, Sabath LD. Endocarditis caused by coagulase-negative staphylococci. Infection $1979 ; 7: 5-9$. 


\section{Clinical Vignette}

4. Geraci JE, Hanson KC, Giuliani ER. Endocarditis caused by coagulase-negative staphylococci. Mayo Clin Proc $1968 ; 43: 420-34$

5. Hamory BH, Parisi JT, Hutton JP. Staphylococcus epidermidis: A significant nosocomial pathogen. Am J Infect Control 1987;15:59-74.

6. Pfaller MA, Herwaldt LA. Laboratory, clinical and epidemiological aspects of coagulase-negative staphylococci. Clin Microbiol Rev 1988;1:281-99.

7. Males BM, Rogers WA Jr, Parisi JT. Virulence factors of biotypes of
Staphylococcus epidermidis from clinical sources. J Clin Microbiol 1975;1:256-61.

8. Sovenir D, Anderson DE Jr, Palpant S, et al. Blood cultures positive for coagulase-negative staphylococci: Antisepsis, pseudobacteremia, and therapy of patients. J Clin Microbiol 1998;36:1923-6.

9. Khatib R, Riederer KM, Clark JA, Khatib S, Briski LE, Wilson FM. Coagulase-negative staphylococci in multiple blood cultures: Strain relatedness and determinants of same-strain bacteremia. J Clin Microbiol 1995;33:816-20. 


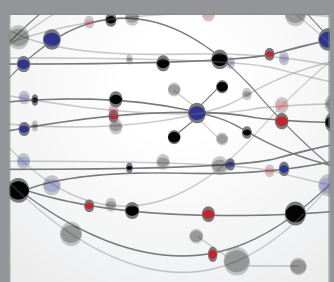

The Scientific World Journal
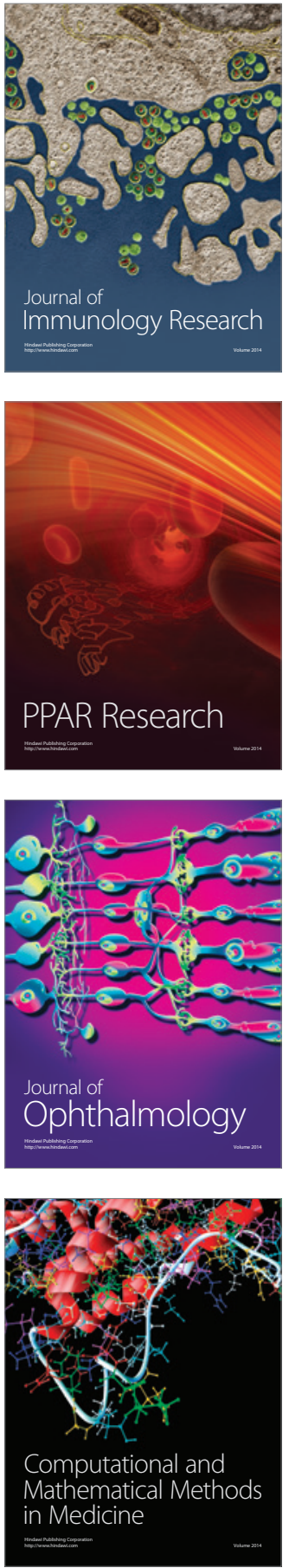

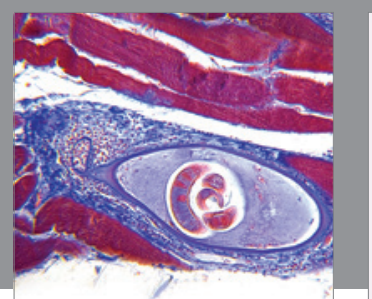

Gastroenterology Research and Practice

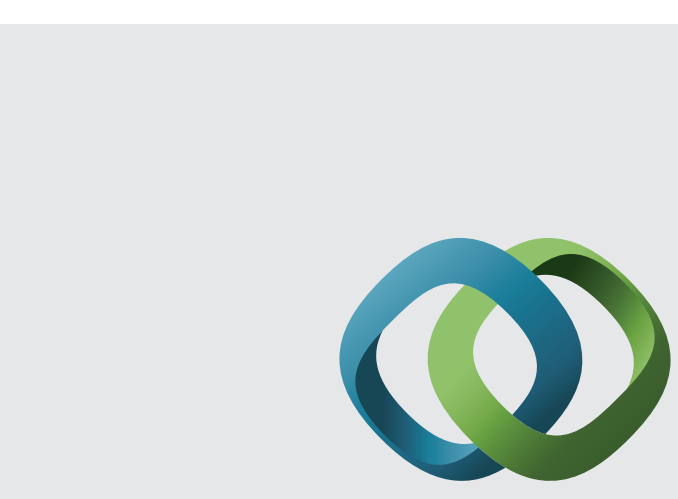

\section{Hindawi}

Submit your manuscripts at

http://www.hindawi.com
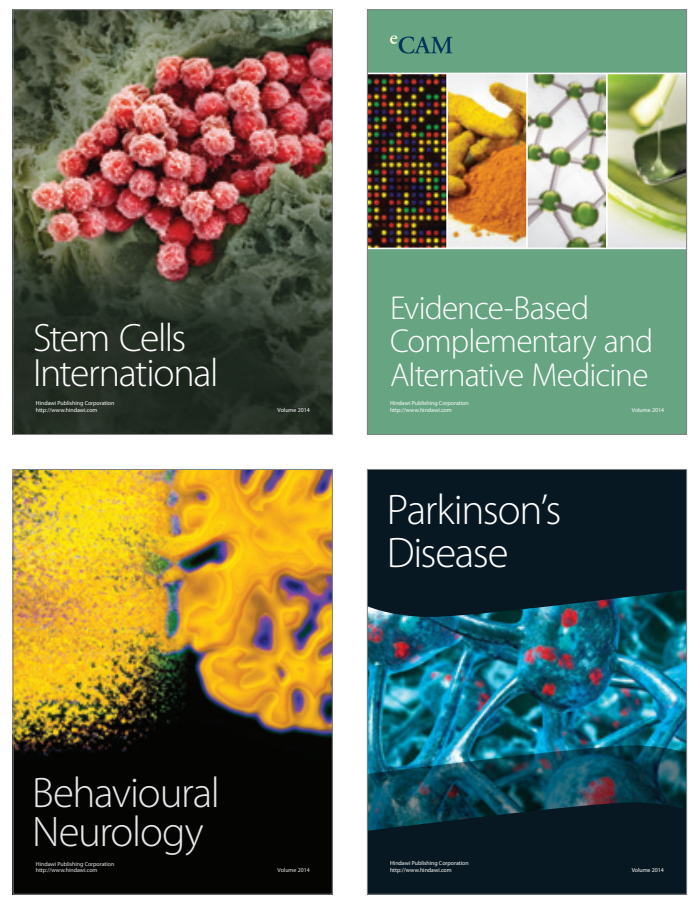
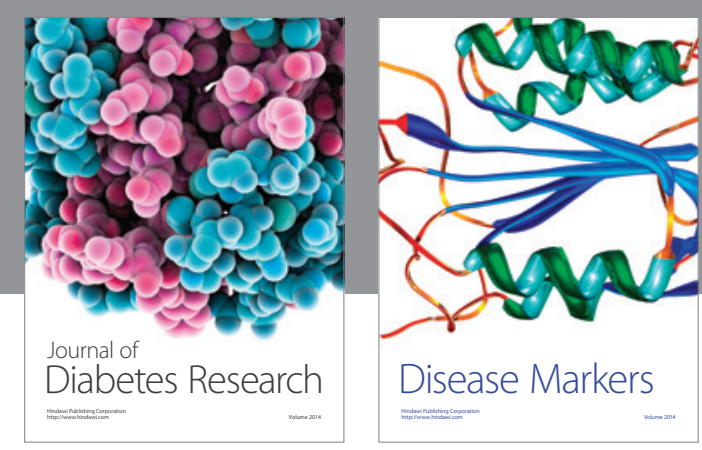

Disease Markers
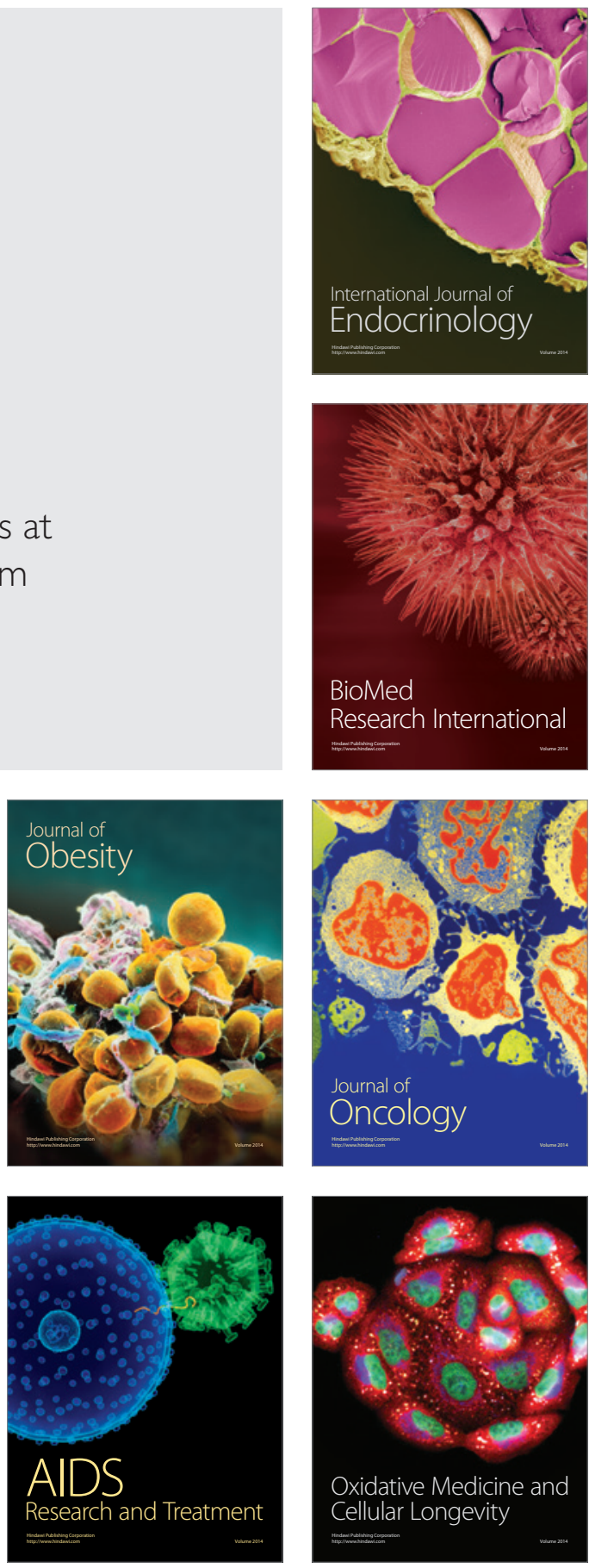